\section{Martian geology}

The Geology of Mars. By T. A. Mutch, R. E. Arvidson, J. W. Head, K. L. Jones and R. S. Saunders. Pp. ix +400 . (Princeton University: Princeton, New Jersey, 1977.) £28.40; \$35.

WRITING a book about our planetary companions in the Solar System is, and has been for over ten years, a hazardous business. The rate of exploration has been such that inevitably parts of such a book are out-of-date even before it arrives on the bookstands; this is because new, important and often revolutionary information is neturned from another exciting space mission. Despite this, and despite the fact that we are in the middle of the highly successful Viking mission to Mars, Thomas Mutch and his colleagues have produced an extremely timely book on the geology of Mars. Essentially, it summarises our knowledge of this planet up to the time of Viking, although it does include pictures and information from the early part of the Viking mission slipped into the book at the last moment. The book is a valuable contribution to planetary studies because it provides (as did Glasstone's The Book of Mars before Mariners 6, 7 and 9) an up-to-date summary of our knowledge of this fascinating planet up to the time of Viking-a useful time to stop and take stock of the situation.

The book opens with two chapters describing early telescopic observations and the history of spacecraft exploration of Mars. This is followed in the third chapter by a summary of the major physiographical provinces of Mars and a discussion of how each of the landforms characteristic of different parts of Mars may have been formed. The main bulk of the book consists of individual chapters on surface processes including impact cratering, volcanism and the action of wind and water, all of which have played a part in producing the martian surface as we see it today. There are also discussions of geophysics, structure and geological history of Mars.

Geologists not involved in planetary studies have often been disappointed with new discoveries from both the Moon and Mercury because so much of the geology of these planets is dominated by impact cratering, a geological process of interest to only a few specialists. This is not true of Mars as, although the ancient southern hemisphere of Mars is dominated by impact cratering, the northern hemisphere and poles show abundant evidence of other processes more familiar to the terrestrial geologist. Large areas have been modified by wind erosion and deposition, and the desert geomorphologist cannot fail to be impressed by the extensive dune fields exposed on the martian surface. Winding channels are indicative of water erosion and the areas around the polar caps show thick sequences of layened rocks. Although volcanism probably played an important part in the evolution of all terrestrial planets, it is only on Mars and Earth that we have seen such a variety of large constructs of high topographical relief. Extensive patterns of rifts and faults show that the tectonic history of Mars has been quite unlike that of the Earth and an understanding of this is essential to developing a general theory for planetary tectonics.

\section{Molecular potential energy surfaces}

Symmetry Rules for Chemical Reactions: Orbital Topology and Elementary Processes. By R. G. Pearson. Pp. ix +548 . (Wiley-Interscience, New York and London, 1976.) $\$ 31 ; £ 17.50$.

THE title of this work is not altogether successful in describing the contents. I do not venture to suggest a better one; but Professor Pearson's concern is with the nature of molecular potential energy surfaces generally, and with their influence on the shapes of molecules as well as on the courses of neactions. His thesis rests on two main principles. The first, due principally to Bader, although certainly used earlier, is that a distortion of a particular symmetry is favoured by the presence of a low-lying electronically excited state of a nelated symmetry. (Perhaps this should be called the 'Bader effect' since the usual 'secondorder Jahn-Teller effect' is a clumsy and inaccurate term.) The distortion may lead a molecule from one postulated structure to another, in which case one obtains an understanding of the shapes of molecules. Walsh's rules are usefully approached in this way. Alternatively, the distortion may initiate a reaction, in which case one obtains an understanding of the relative ease of alternative reaction paths.

The second principle is that of "orbital correlation" or "conservation of orbital symmetry": the symmetry species of the molecular orbitals in reactants and products must match. If they do not, then at least one bonding orbital in the reactants correlates with an anti-bonding orbital in the products,
The authors of this book do an excellent job of summarising and discussing critically our knowledge of all these subjects. They also briefly compare Mars with the other planets, including Earth, showing how all the solid planets in the Solar System are important field areas for Earth-bound geologists.

This book will not only be a useful reference book for planetary geologists but provides an easily readable and well illustrated review for the non-specialist. For a student it is an important text, although sadly the price is high (especially the sterling equivalent); and students may have to rely on library copies. J. E. Guest

J. E. Guest is Lecturer in the Department of Physics and Astronomy at University College, London, UK.

which in turn implies a high activation energy. In the application of these principles, a third is used: the predicted behaviour depends on the "orbital topology"- $\rightarrow$ the nodal structure of the molecular orbitals-which is not significantly modified even if the symmetry is broken by irnelevant changes to the molecule. Consequently symmetry-based methods may often be used where no formal symmetry is present.

Professor Pearson expounds these principles with admirable clarity and numerous examples. The Jahn-Teller selection rule is incorrectly stated (the correct version is subsequently used, but with no explanation of the notation for the symmetrised square) and the account of the first-order Jahn-Teller effect contains some very dubious remarks. Fortunately, the first-order Jahn-Teller effect is of only peripheral importance in the later development. A more general criticism is that the magnitudes of the effects cited are not always shown to be sufficient to account quantitatively for the observations. For example, the dissociation energy of ozone is $1 \mathrm{eV}$, whereas that of sulphur dioxide is $5.5 \mathrm{eV}$; this is explained by the fact that the relevant excited state in ozone is at $4.7 \mathrm{eV}$, whereas the corresponding state in sulphur dioxide is at $5.3 \mathrm{eV}$. The difference is in the right direction but the numerical values are unconvincing.

For the most part, however, Professor Pearson approaches his subject canefully, critically and thoroughly. This is a stimulating and valuable book, and an important contribution to a rapidly developing field of research.

A. J. Stone

A. J. Stone is an Assistant Director of Research in Theoretical Chemistry at the University of Cambridge, UK. 